\title{
Genetic Mutation of SARS-CoV-2 during Consecutive Passages in Permissive Cells
}

\author{
Ying Chen ${ }^{1,2} \cdot$ Mei-Qin Liu ${ }^{1,2} \cdot$ Yun Luo ${ }^{1,2} \cdot$ Ren-Di Jiang ${ }^{1,2} \cdot$ Hao-Rui $\mathrm{Si}^{1,2} \cdot$ Yan Zhu ${ }^{1} \cdot \mathrm{Bei} \mathrm{Li}^{1} \cdot$ \\ Xu-Rui Shen ${ }^{1,2} \cdot$ Hao-Feng Lin $^{1,2} \cdot$ Kai Zhao ${ }^{1,2} \cdot$ Ben Hu ${ }^{1} \cdot$ Zheng-Li Shi $^{1} \cdot$ Xing-Lou Yang $^{1}$ (D)
}

Received: 8 December 2020 / Accepted: 15 March 2021 / Published online: 26 April 2021

(c) Wuhan Institute of Virology, CAS 2021

Dear Editor,

The ongoing COVID-19 disease, caused by severe acute respiratory syndrome coronavirus 2 (SARS-CoV-2), has led to over 112 million confirmed cases and 2.4 million deaths in more than 220 countries as of 25 February 2021 (WHO 2021). Hospital-admitted patients show clinical features including fever, dry cough, fatigue, dyspnea, lymphopenia, and pneumonia with radiological groundglass lung opacities (Huang et al. 2020; Guan et al. 2020). SARS-CoV-2 was quickly isolated and could be detected in clinical samples, such as nasopharyngeal swabs, sputum, alveolar lavage fluid, and feces, as well as occasionally in seminal fluid and tears among other sources (Bhat et al. 2020; Li et al. 2020), which means that it can infect a variety of human tissues and organs.

SARS-CoV-2 is classified as a SARS-related coronavirus (SARSr-CoV) and has $79.5 \%$ and $96.2 \%$ identity, respectively, with SARS-CoV and a bat SARSr-CoV (RaTG13) at the full-genome level, indicating that it could have also originated from bats (Hu et al. 2017; Zhou et al. 2020). Thereafter, two SARSr-CoV strains, which have $85 \%$ and $91 \%$ nt genomic sequence identities with SARS$\mathrm{CoV}-2$, were isolated from Malayan pangolins (Lam et al. 2020; Xiao et al. 2020). To date, there is no evidence to support the direct bat or pangolin origin of SARS-CoV-2. It

Supplementary Information The online version contains supplementary material available at https://doi.org/10.1007/s12250021-00384-w.

Xing-Lou Yang

yangxl@wh.iov.cn

1 CAS Key Laboratory of Special Pathogens and Biosafety, Wuhan Institute of Virology, Center for Biosafety MegaScience, Chinese Academy of Sciences, Wuhan 430071, China

2 University of the Chinese Academy of Sciences, Beijing 100049, China was reported that this virus exhibits tropism towards a wide range of cells and hosts, and a series of mutations were found in person-to-person and in vitro passages (Liu et al. 2020). To further investigate the genetic susceptibility of SARS-CoV-2 during the passage on different cells, we identified nine cell lines susceptible to this virus among 30 different transformed or primary cell lines; then the different SARS-CoV-2 strains isolated on Vero E6 and Huh-7 from a same patient bronchoalveolar lavage fluid (BALF) were serially passaged in Vero E6, Huh-7 and Caco-2 cell lines and continuously monitored.

Cell susceptibility was determined by an indirect immunofluorescence assay (IFA) targeting the nucleocapsid (N) protein of SARS-CoV-2 (Zhou et al. 2020). Briefly, the SARS-CoV-2 isolated on Vero E6 cell line was cultured and titrated on Vero E6 cells. Then, different cell lines were seeded in 24 well plates and infected with SARS-CoV-2 at multiplicity of infection 1 . The infected cells were washed with PBS and fixed with $4 \%$ paraformaldehyde at $24 \mathrm{~h}$ post-infection (hpi) overnight and then treated with TritonX-100 for cell membrane perforation. The treated cells were incubated at $4{ }^{\circ} \mathrm{C}$ with a cross-reactive viral $\mathrm{N}$ antibody (rabbit anti-SARSr-CoV Rp3 $\mathrm{N}$ protein polyclonal antibody, 1:1000, made inhouse). After washing with PBS, cells were incubated with Cy3-conjugated goat-anti-rabbit IgG (1:200, Abcam, ab6939) secondary antibody for $1 \mathrm{~h}$, and then, the nuclei were stained with DAPI (1:100, Beyotime). Then, the cells were observed under an AMF4300 EVOS FL cell Imaging System (Life Technologies, Carlsbad, CA, USA). Among the 30 tested cell lines, nine were positive by IFA (Fig. 1A, Supplementary Table S1). They comprised six human cell lines from various human organs, including Calu-3 (lung), HEp-2 (larynx), Huh-7 and Hep G2 (liver), Caco-2 (colon), and $\mathrm{HaCaT}$ (skin), two non-human primate cell lines including Vero E6 (kidney) and LLC-MK2 (kidney), and one swine cell line ST (testicle). Based on the expression of $\mathrm{N}$ protein, Vero E6, Caco-2, Huh-7, and Calu-3 cells were 
A

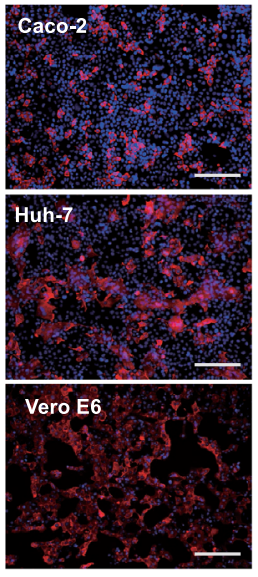

B

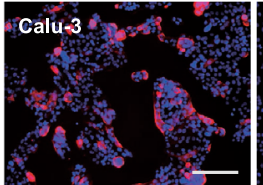

$\mathrm{Hep} \mathrm{C2}_{2}$
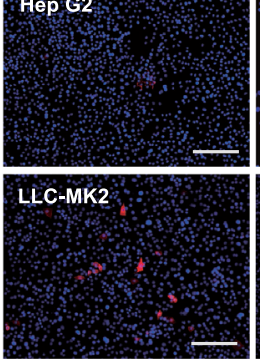
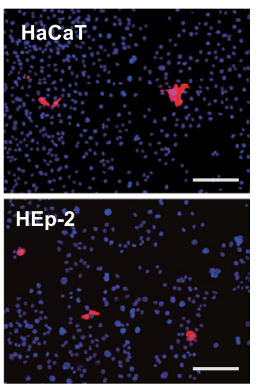

ST
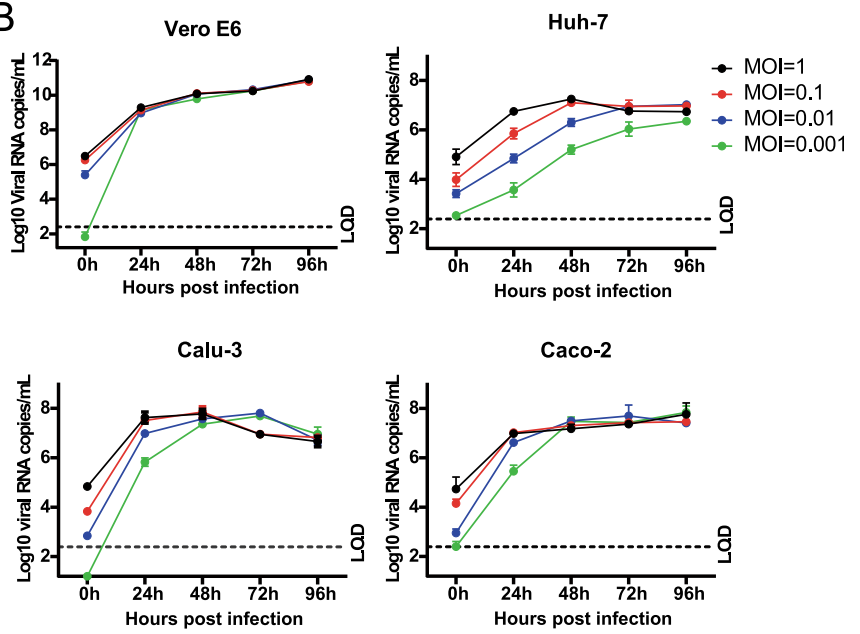

C

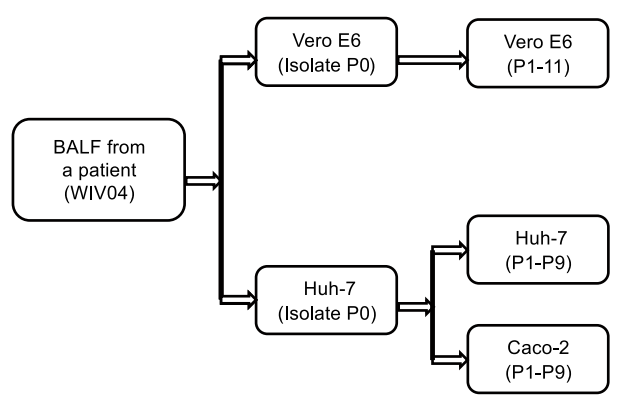

\begin{tabular}{ll}
\hline Passage & Gene mutation(nt, aa) \\
\hline Isolate P0 & ORF1ab, T565C, S-S \\
(Compare to WIV04) & ORF1ab, C17825T, T-I
\end{tabular}

(Compare to WIV04) $\quad$ 3' UTR, G29573A

P1-P11 P2-P4, Spike, T21784A, N-K

(Compare to P0 in Vero E6) P5, Spike, T21784A, N-K; Spike, G23607A, R-Q

6-P11, Spike, T21784A, N-K; Spike, G23607A, R-Q; Spike, C23525T, H-Y

Isolate P0 ORF1ab, G2801A, D-Y

(Compare to WIV04) ORF1ab, C10868T, L-L

P1-P9

(Compare to $\mathrm{PO}$ in Huh-7) No mutation

P1-P9 P1-P5, no mutation

$\begin{array}{ll}\text { (Compare to } \mathrm{P} 0 \text { in Huh-7) } & \mathrm{P} 6-\mathrm{P} 9, \text {, Sp mutation } \\ \end{array}$

D

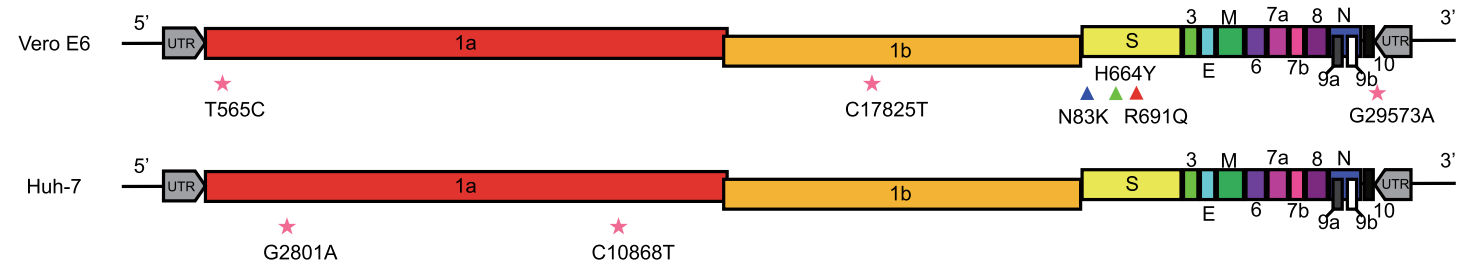

Caco-2

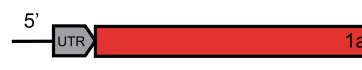

C10868T
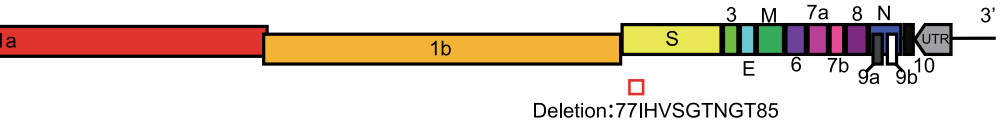

Fig. 1 Susceptibility, replication efficiency, and mutations of SARSCoV-2 in different cell lines. A Expression of N protein detected by immunofluorescence assays (IFAs) at $24 \mathrm{~h}$ post-infection (hpi) in SARS-CoV-2-infected cell lines, scale bar: $200 \mu \mathrm{m}$. B Viral loads detected by qRT-PCR in the culture supernatants of Calu-3, Caco-2, Huh-7, and Vero E6 cells infected with SARS-CoV-2 in duplicate at MOIs of $0.001,0.01,0.1$, and 1 . Supernatants were harvested at 0,24 ,

more susceptible than other cells at $24 \mathrm{hpi}$, and HaCaT, ST and Hep G2 cells exhibited lower susceptibility.

To further compare the viral replication efficiency in different cell lines that were found to be highly susceptible to SARS-CoV-2 infection, we infected Calu-3, Huh-7, Caco-2, and Vero E6 cell lines with MOIs of 0.001, 0.01, 0.1 , and 1 (Fig. 1B). Viral RNA was extracted from supernatants of infected cells at different time points using
48, 72, and 96 hpi. C Passage scheme and mutation information of SARS-CoV-2. D Schematic diagram of mutations in SARS-CoV-2 after isolated and passaged in Vero E6, Huh-7, and Caco-2 cell lines. The mutations were observed after the isolation (pink star), 2nd (blue triangle), 5th (red triangle or rectangle), and 6th (green triangle) passage.

the QIAamp 96 Virus QIAcube HT Kit (Qiagen, Germany). The viral RNA copies in the supernatant were quantified with the HiScript no. II One Step qRT-PCR SYBR no. Green Kit (Vazyme, Nanjng, China) as we previously described (Zhou et al. 2020). The primers based on the SARS-CoV-2 $S$ gene were designed as: RBD-qF1: 5'-CAATGGTTTAACAGGCACAGG-3'; RBD-qR1: 5' CTCAAGTGTCTGTGGATCACG- $3^{\prime}$. The viral loads of 
SARS-CoV-2 all increased more than $4 \log$ values and showed increasing trends with an escalating MOI. Vero E6 cells showed the highest susceptibility to SARS-CoV-2, as up to $10^{11}$ RNA viral copies $/ \mathrm{mL}$ supernatant were detected at an MOI of 1 at 96 hpi. Huh-7, Caco-2, and Calu-3 cells showed equivalent susceptibility to SARS-CoV-2 with up to $10^{7}$ viral RNA copies $/ \mathrm{mL}$. The viral loads in Calu-3 cells peaked at 48 hpi with MOIs of 1 and 0.1 or at 72 hpi with MOIs of 0.01 and 0.001 . The viral loads in Huh-7 cells peaked at 48 hpi with MOIs of 1 and 0.1 or at 96 hpi with MOIs of 0.01 and 0.001 . Meanwhile, the viral loads in Caco-2 cells peaked at 72 hpi with an MOI of 0.01 or at 96 hpi with MOIs of $1,0.1$, and 0.001 .

Previous studies have shown that mutations in viral genes occur during virus passage in Vero and Vero E6 cells (Liu et al. 2020; Vankadari 2020). We further investigated whether a primary isolate could be efficiently maintained in other susceptible cell lines. SARS-CoV-2 WIV04 genome was sequenced from a patient BALF sample (Zhou et al. 2020). Virus was isolated successfully in Vero E6 and Huh-7 cells from the same BALF sample; three and two mutations were observed in the virus genomes after isolation in Vero E6 and Huh-7 cells, respectively, compared to SARS-CoV-2 WIV04 genome (Fig. 1C, 1D). These results suggest that there might be adaptations for SARS-CoV-2 in different cell lines. The SARS-CoV-2 isolate from Vero E6, was serially passaged in Vero E6 cell line for 11 passages; while the SARS-CoV-2 isolate from Huh-7 was serially passaged in Huh-7 and Caco-2 for 9 passages, respectively (Fig. 1C, 1D). Supernatants were collected from every passage of each cell line, and then, next-generation sequencing (MGISEQ-2000RS FAST, Shenzhen MGI Tech Co., Ltd.) via the direct RNA sequencing was conducted to investigate the dynamics of mutations. Additional mutations were observed in N83K, R691Q, and $\mathrm{H} 664 \mathrm{Y}$ of the spike protein from the P2, P5, and P6 passages in Vero E6, respectively. However, no more mutations were observed in Huh-7 cells over 9 consecutive passages. Notably, a 27-nucleotide deletion occurred in the spike protein resulting in a deletion of nine amino acids (77-85 IHVSGTNGT) when SARS-CoV-2 was passaged to the P6 generation in Caco-2 cells, and this deletion variant was stable in the following 3 passages.

In our study, we investigated the susceptibility, cell tropism, and viral replication efficiency of SARS-CoV-2. Results showed that nine of thirty cell lines derived from various tissues of humans (including lung, liver, colon, larynx, and skin), monkey (kidney), and swine (testicle) were susceptible to SARS-CoV-2. The virus replicated efficiently in human cell lines (Huh-7, Calu-3, Caco-2) and non-human primate cells (Vero E6) but less so in swine cells. In a previous study, it was reported that five human cell lines, including Calu-3, Caco-2, Huh-7, 293T, and
U251, and six non-human cell lines, including Vero E6, FRhK4, LLC-MK2, CRFK, RK-13, and PK-15, were susceptible to SARS-CoV-2 (Chu et al. 2020). In our study, we showed that three additional human cell lines, including HEp2 (larynx), Hep G2 (liver), and HaCaT (skin), are slightly susceptible to SARS-CoV-2 infection. Our study, together with others, demonstrates the wide cell tropism of SARS-CoV-2 in human and non-human primates in vitro. Meanwhile, the swine cell line ST (testicle) showed low susceptibility. Unexpectedly, none of the tested bat cell lines supported SARS-CoV-2 replication. Previously, we found that HeLa cells expressing ACE2 from Rhinolophus sinicus could support SARS-CoV-2 entry. This inconsistency might be due to the low or undetectable expression of ACE2 in immortal bat cell lines. We also investigated the susceptibility of other animal cell lines, including those from canine, mink, hamster, rat, and mouse; none of these cell lines could be infected with SARS-CoV-2. A possible explanation for this is that these cells do not express or have lower expression of ACE2 proteins or that SARSCoV-2 does not use ACE2 of these cells as the cellular entry receptor (Zhao et al. 2020).

The viral replication efficiency results showed that the Vero E6 cell line was more susceptible to SARS-CoV-2 than the Caco-2, Huh-7, and Calu-3 cell lines. Our results verified that the Vero E6 cell line is suitable for viral amplification. However, the results of our group and others suggested that the spike glycoprotein of SARS-CoV-2 is more likely to mutate during culture in this cell line. Most strikingly, no mutations were observed in Huh-7 cells during 10 consecutive passages, indicating that the virus is more stable in this cell line, which indicates that they could be applicable for investigations of pathogenicity and pathogenesis. However, as a human intestinal cell line, culture in Caco-2 cells resulted in a 27-nt deletion in the NTD of the spike glycoprotein, whereas a mutant with this deletion was not found in human clinical specimens. These results suggest that the spike glycoprotein of SARS-CoV-2 is more likely to mutate during in vitro passage, which is consistent with the reported outcomes of person-to-person transmission (Gong et al. 2020). It remains largely unknown whether mutations acquired during in vitro passages additionally affect viral intrinsic characteristics. Continuous monitoring of SARS-CoV-2 could benefit not only biological studies of this virus but also vaccine development in the future.

Acknowledgements We acknowledge Microorganisms \& Viruses Culture Collection Center of Wuhan Institute of Virology for provided cells. This work was jointly supported by the China Natural Science Foundation (32070180 to X.-L.Y., 31770175 to Z.-L.S.), Strategic Priority Research Program of the Chinese Academy of Sciences (XDB29010101 to Z.-L.S.), and a Youth innovation promotion association of CAS (2019328 to X.-L.Y.). 


\section{Compliance with Ethical Standards}

Conflict of interest The authors declare that they have no conflict of interest.

Animal and Human Rights Statement This article does not contain any studies with human and animal subjects performed by any of the authors.

\section{References}

Bhat TA, Kalathil SG, Bogner PN, Blount BC, Goniewicz ML, Thanavala YM (2020) An animal model of inhaled vitamin E acetate and EVALI-like lung injury. $\mathrm{N}$ Engl $\mathrm{J}$ Med 382:1175-1177

Chu H, Chan JF-W, Yuen TT-T, Shuai H, Yuan S, Wang Y, Hu B, Yip CC-Y, Tsang JO-L, Huang X, Chai Y, Yang D, Hou Y, Chik KK-H, Zhang X, Fung AY-F, Tsoi H-W, Cai J-P, Chan W-M, Ip JD, Chu AW-H, Zhou J, Lung DC, Kok K-H, To KK-W, Tsang OT-Y, Chan K-H, Yuen K-Y (2020) Comparative tropism, replication kinetics, and cell damage profiling of SARS-CoV-2 and SARS-CoV with implications for clinical manifestations, transmissibility, and laboratory studies of COVID-19: an observational study. Lancet Microbe 1:e14-e23

Gong Z, Zhu JW, Li CP, Jiang S, Ma LN, Tang BX, Zou D, Chen ML, Sun YB, Song SH, Zhang Z, Xiao JF, Xue YB, Bao YM, Du ZL, Zhao WM (2020) An online coronavirus analysis platform from the National Genomics Data Center. Zool Res 41:705-708

Guan WJ, Ni ZY, Hu Y, Liang WH, Ou CQ, He JX, Liu L, Shan H, Lei CL, Hui DSC, Du B, Li LJ, Zeng G, Yuen KY, Chen RC, Tang CL, Wang T, Chen PY, Xiang J, Li SY, Wang JL, Liang ZJ, Peng YX, Wei L, Liu Y, Hu YH, Peng P, Wang JM, Liu JY, Chen Z, Li G, Zheng ZJ, Qiu SQ, Luo J, Ye CJ, Zhu SY, Zhong NS, China Medical Treatment Expert Group for C (2020) Clinical characteristics of coronavirus disease 2019 in China. N Engl J Med 382:1708-1720

Hu B, Zeng LP, Yang XL, Ge XY, Zhang W, Li B, Xie JZ, Shen XR, Zhang YZ, Wang N, Luo DS, Zheng XS, Wang MN, Daszak P, Wang LF, Cui J, Shi ZL (2017) Discovery of a rich gene pool of bat SARS-related coronaviruses provides new insights into the origin of SARS coronavirus. PLoS Pathog 13:e1006698

Huang C, Wang Y, Li X, Ren L, Zhao J, Hu Y, Zhang L, Fan G, Xu J, Gu X, Cheng Z, Yu T, Xia J, Wei Y, Wu W, Xie X, Yin W, Li H, Liu M, Xiao Y, Gao H, Guo L, Xie J, Wang G, Jiang R, Gao Z, Jin Q, Wang J, Cao B (2020) Clinical features of patients infected with 2019 novel coronavirus in Wuhan, China. Lancet 395:497-506

Lam TT, Jia N, Zhang YW, Shum MH, Jiang JF, Zhu HC, Tong YG, Shi YX, Ni XB, Liao YS, Li WJ, Jiang BG, Wei W, Yuan TT, Zheng K, Cui XM, Li J, Pei GQ, Qiang X, Cheung WY, Li LF, Sun FF, Qin S, Huang JC, Leung GM, Holmes EC, Hu YL, Guan Y, Cao WC (2020) Identifying SARS-CoV-2-related coronaviruses in Malayan pangolins. Nature 583:282-285

Li D, Jin M, Bao P, Zhao W, Zhang S (2020) Clinical characteristics and results of semen tests among men with coronavirus disease 2019. JAMA Netw Open 3:e208292

Liu Z, Zheng H, Lin H, Li M, Yuan R, Peng J, Xiong Q, Sun J, Li B, Wu J, Yi L, Peng X, Zhang H, Zhang W, Hulswit RJG, Loman N, Rambaut A, Ke C, Bowden TA, Pybus OG, Lu J (2020) Identification of common deletions in the spike protein of severe acute respiratory syndrome coronavirus 2. J Virol 94:e00790-20

Vankadari N (2020) Overwhelming mutations or SNPs of SARSCoV-2: a point of caution. Gene 752:144792

WHO (2021) Coronavirus disease (COVID-2019) situation reports. https://www.who.int/emergencies/diseases/novel-coronavirus2019/situation-reports

Xiao K, Zhai J, Feng Y, Zhou N, Zhang X, Zou JJ, Li N, Guo Y, Li X, Shen X, Zhang Z, Shu F, Huang W, Li Y, Zhang Z, Chen RA, Wu YJ, Peng SM, Huang M, Xie WJ, Cai QH, Hou FH, Chen W, Xiao L, Shen Y (2020) Isolation of SARS-CoV-2-related coronavirus from Malayan pangolins. Nature 583:286-289

Zhao X, Chen D, Szabla R, Zheng M, Li G, Du P, Zheng S, Li X, Song C, Li R, Guo J-T, Junop M, Zeng H, Lin H (2020) Broad and differential animal ACE2 receptor usage by SARS-CoV-2. J Virol 94:e00942-20

Zhou P, Yang XL, Wang XG, Hu B, Zhang L, Zhang W, Si HR, Zhu Y, Li B, Huang CL, Chen HD, Chen J, Luo Y, Guo H, Jiang RD, Liu MQ, Chen Y, Shen XR, Wang X, Zheng XS, Zhao K, Chen QJ, Deng F, Liu LL, Yan B, Zhan FX, Wang YY, Xiao GF, Shi ZL (2020) A pneumonia outbreak associated with a new coronavirus of probable bat origin. Nature 579:270-273 